\title{
Detailed Star-Formation Histories of Nearby Dwarf Irregular Galaxies using HST $^{1}$
}

\author{
Eline Tolstoy \\ European Southern Observatory, Garching, Germany
}

\begin{abstract}
Unless the nearby universe is subtly anomalous, it should contain a relatively normal selection of galaxies whose histories are representative of field galaxies in general throughout the Universe. We can therefore take advantage of our ability to resolve the nearby galaxies into individual stars to directly, and accurately, measure star formation histories. The star formation histories are determined from model-fitting analysis, based on stellar evolution tracks, of colour-magnitude diagrams. The most accurate information on star formation rates extending back to the earliest epochs can be obtained from the structure of the main sequence. However, the oldest main sequence turnoffs are very faint, and it is often necessary to use the more evolved stars to infer the star formation history. These can then be compared with the properties of galaxies seen over a large range of lookback times at moderate to high redshifts. There is considerable evidence that the faint blue galaxies seen in large numbers in cosmological redshift surveys are the progenitors of the small late-type irregular galaxies seen in copious numbers in the Local Group, and we concentrate on these here.
\end{abstract}

\section{Introduction}

The study of resolved stellar populations provides a powerful tool to follow galaxy evolution directly in terms of physical parameters such as age (star formation history, SFH), chemical composition and enrichment history, initial mass function, environment, and dynamical history of the system. Photometry of individual stars in at least two filters and the interpretation of Colour-Magnitude Diagram (CMD) morphology gives the least ambiguous and most accurate information about variations in star formation rate $(s f r)$ within a galaxy back to the oldest stars (see Fig. 1). Some of the physical parameters that affect a CMD are strongly correlated, such as metallicity and age, since successive generations of star formation may be progressively enriched in the heavier elements. Careful, detailed CMD analysis is a proven, uniquely powerful approach (e.g., Tosi et al. 1991; Tolstoy \& Saha 1996; Aparicio et al. 1996; Mighell 1997; Dohm-Palmer et al. 1997a,b; Hurley-Keller et al. 1998; Gallagher et al. 1998; Tolstoy et al.

\footnotetext{
${ }^{1}$ Based on observations with the NASA/ESA Hubble Space telescope, obtained at the Space Telescope Science Institute, which is operated by the Association of Universities for Research in Astronomy (AURA), Inc., under NASA contract NAS 5-26555.
} 
1998; Tolstoy 1998) that benefits enormously from high spatial resolution, high quality imaging. HST has led the way in recent years, and we are optimistic that ground based telescopes capable of achieving excellent seeing, such as VLT, will also provide much useful data, especially in the blue, and in relatively sparse stellar systems such as dwarf irregular (dI) galaxies.

The small $\mathrm{dI}$ and $\mathrm{dSph}$ galaxies are considered the most likely connection to higher redshift, late type evolving systems, which makes them the most interesting to look at when trying to make a connection to the high redshift universe. In the LG they appear to exhibit a wide variety of SFHs. These results have affected our understanding of galaxy formation and evolution by demonstrating the importance of episodic star formation in nearby low mass galaxies. The larger galaxies in the LG have evidence of sizeable old halos, which appear to represent the majority of star formation in the LG by mass, although the problems distinguishing between effects of age and metallicity in a CMD result in a degree of uncertainty in the exact age distribution in these halos. High quality, deep imaging is the only way of extending detailed SFH studies past the Galaxy and its satellites, enabling us to obtain a picture of the fossil record of star formation in galaxies of various types and sizes, and to identify both commonalities and differences in their SFH across the LG to be able to make the comparison with cosmological surveys (Tolstoy 1998) more accurate.

The Local Group (LG) will provide a picture of the global star formation properties of galaxies with a wide variety of mass, metallicity, gas content etc., (e.g., Mateo 1998) and will make a sample that ought to reflect the SFH of the Universe which can be compared to high redshift surveys (e.g., Madau et al. 1998). Initial comparisons suggest these different approaches do not yield the same results (Tolstoy 1998; Fukugita et al. 1998), but the errors are large due to the lack of detailed SFHs of nearby galaxies. Careful determinations of the complete SFH of nearby galaxies is thus extremely important for understanding star formation properties of nearby galaxies in detail and also to make the connection to high-redshift studies. It is still not clear if bursting star formation is common enough in LG dIs to account for a large population of compact emission line galaxies at intermediate redshifts. If the conclusions of Madau et al. and the SFH of the LG still do not agree then it will be of critical importance for the field of high redshift galaxy research that this discrepancy is understood. It might be that redshift surveys are strongly biased towards low mass dI galaxies undergoing bursts of star formation, and thus they are not accurate indicators of the dominant mode (by mass) of star formation in the Universe.

\section{Colour-Magnitude Diagram Analysis}

Stellar evolution theory provides a number of predictions, based on relatively well understood physics, of features expected in CMDs for different age and metallicity stellar populations (see Fig. 1). There are a number of clear indicators of varying $s f r s$ at different times which can be combined to obtain a very accurate picture of the entire SFH of a galaxy. 


\subsection{Main Sequence Turnoffs (MSTOs)}

If we can obtain deep enough exposures of the resolved stellar populations in nearby galaxies we can obtain the unambiguous age information that comes from the luminosity of MSTOs. MSTOs can clearly distinguish between bursting star formation and quiescent star formation, (e.g., Hurley-Keller et al. 1998). The age resolution that is possible does vary, becoming coarser going back in time. Our ability to disentangle the variations in $s f r$ from MSTOs also depends upon the the intensity of the past variations and how long ago they occured. For ages less than about $1.5 \mathrm{Gyr}$ it is possible to have detailed resolution on the 10-100 Myr time scales. As can be seen in Fig. 1, the ages begin to crowd together for older populations. Beyond about $8 \mathrm{Gyr}$ ago the age resolution is on the scale of roughly a Gyr. This of course means that a very short high intensity burst of star formation in this period will be indistinguishable from a lower $s f r$ but longer lasting epoch of star formation. As will be described in the following sections, however, there are other indicators in a CMD which help to narrow down the range of possible SFHs.

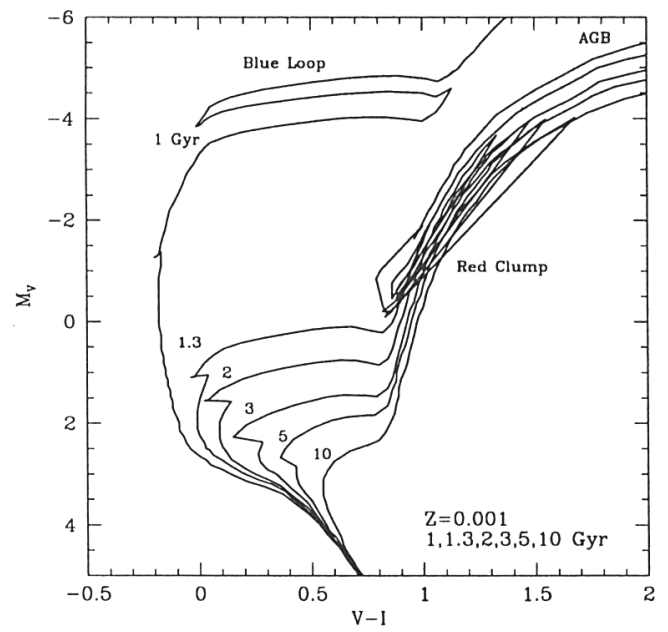

Figure 1. Isochrones for a single metallicity $(Z=0.001)$ and a range of ages (from Bertelli et al. 1994), marked in Gyr, at the MSTOs. Isochrones were designed for single age globular cluster populations and are best avoided in the interpretation of composite populations, which can best be modeled using Monte-Carlo techniques (e.g., Tolstoy 1996).

\subsection{The Core-Helium Burning Blue Loop Stars (BLs)}

Stars of certain metallicity and mass go on "Blue Loop Excursions", after they ignite $\mathrm{He}$ in their core (see Fig. 1). Stars in the BL phase can be several magnitudes brighter than when on the MS. The luminosity of a BL star is fixed for a given age. Subsequent generations of BL stars do not overlie each other as they do on the MS. The shape and mass at which these "loops" are seen in a CMD are a strong function of metallicity and age, in the sense that the lower the metallicity of the galaxy the further back in time an accurate SFH can easily be determined, which makes them especially useful for low metallicity systems such as dI galaxies. (e.g., Dohm-Palmer et al. 1997a,b, 1998; Tolstoy et al. 1998). 


\subsection{The Red Giant Branch (RGB)}

The RGB is a very bright evolved phase of stellar evolution, where the star is burning $\mathrm{H}$ in a shell around its He core. For a given metallicity the RGB red and blue limits are given by the young and old limits (respectively) of the stars populating it (for ages $\gtrsim 1 \mathrm{Gyr}$ ). As a stellar population ages the RGB moves to the red, and for constant metallicity, the blue edge is determined by the age of the oldest stars. However, increasing the metallicity of a stellar population will also produce exactly the same effect as aging, and also makes the RGB redder. This is the age-metallicity degeneracy problem. The result is that if there is metallicity evolution within a galaxy, it can be impossible to uniquely disentangle effects due to age and metallicity on the basis of the optical colours of the RGB alone, especially in low metallicity systems such as dI galaxies (cf. Da Costa 1997)

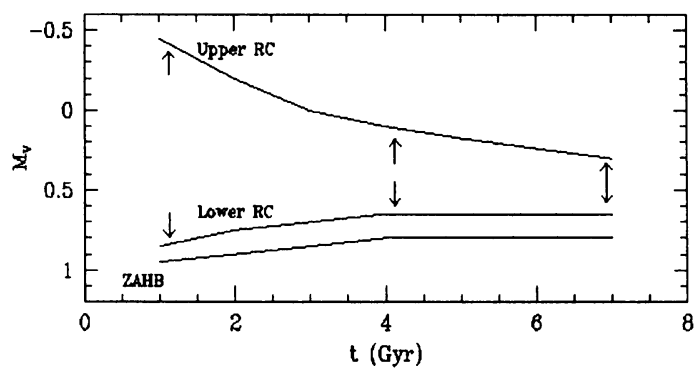

Figure 2. In the top panel is plotted the magnitude of the upper and lower edge of the $\mathrm{RC}$ versus age, in Gyr, from Caputo et al. (1995). This shows the variation in the $e x$ tent in $\mathrm{M}_{V}$ of a $\mathrm{RC}$ with age, for a metallicity of $Z=0.0004$. It can be clearly see that this extent is a strong function of the age of the stellar population. Also plotted is $\mathrm{M}_{V}$ of the zero age $\mathrm{HB}$ against age. In the bottom panel are plotted the results of running a series of Monte-Carlo simulations using stellar evolution models at $Z=0.0004$ (from Fagotto et al. 1994) and counting the number of RC and RGB stars in the same part of the diagram, and thus we determine the expected ratio of $\mathrm{RC} / \mathrm{RGB}$ stars versus age.

\subsection{The Red Clump/Horizontal Branch (RC/HB)}

Red Clump (RC) stars and their lower mass cousins, Horizontal Branch (HB) stars are core helium-burning stars, and their luminosity varies depending upon age, metallicity and mass loss (e.g., Caputo et al. 1995). The extent in luminosity of the RC can be used to estimate the age of the population that produced it, as shown in the upper panel of Fig. 2. This age measure is independent of absolute magnitude and hence distance, and indeed these properties can be used to determine an accurate distance measure on the basis of the RC (e.g., Cole 1998).

The classical RC and RGB appear in a population at about the same time ( 0.9-1.5 Gyr, depending on model details), where the RGB are the progenitors of the $\mathrm{RC}$ stars. The lifetime of a star on the $\mathrm{RGB}, t_{\mathrm{RGB}}$, is a decreasing function 
of $\mathrm{M}_{\mathrm{star}}$, but the lifetime in the $\mathrm{RC}, t_{\mathrm{RC}}$ is roughly constant. Hence the ratio, $t_{\mathrm{RC}} / t_{\mathrm{RGB}}$, is a decreasing function of the age of the dominant stellar population in a galaxy, and the ratio of the numbers of stars in the $\mathrm{RC}$, and the $\mathrm{HB}$, to the number of RGB is sensitive to the SFH of the galaxy (Tolstoy et al. 1998; Han et al. 1997). Thus, the higher the ratio, $N(R C) / N(R G B)$, the younger the dominant stellar population in a galaxy, as shown in the lower panel of Fig. 2 .

The presence of a large $\mathrm{HB}$ population on the other hand (indicated by a high $\mathrm{N}(\mathrm{HB}) / \mathrm{N}(\mathrm{RGB})$ or even $\mathrm{N}(\mathrm{HB}) / \mathrm{N}(\mathrm{MS})$, is caused by a predominantly much older (>10 Gyr) stellar population in a galaxy. The HB is the brightest indicator of very lowest mass (hence oldest) stellar populations in a galaxy.

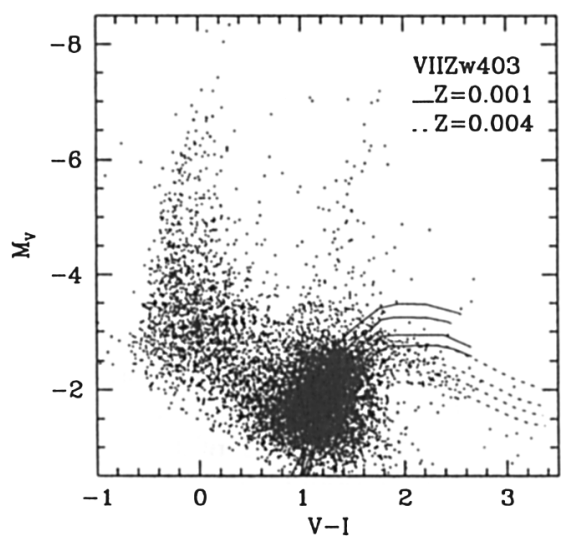

Figure 3. EAGB isochrones for metallicities, $Z=0.001$ and $Z=0.004$, are shown superposed on the observed CMD of VII Zw 403 . For each metallicity the isochrones are for populations of ages $1.3,2,3$, and 5 Gyr, with the youngest isochrone being the brightest. This shows the potential discriminant between the age and metallicity of older populations, if the models could be calibrated.

\subsection{The Extended Asymptotic Giant Branch (EAGB)}

The temperature and colour of the EAGB stars in a galaxy are determined by the age and metallicity of the population they represent. However, there remain a number of uncertainties in the comparison between the models and the data (e.g., Gallart et al. 1994; Lynds et al. 1998). It is very important that more work is done to calibrate these very bright indicators of past star formation events. In Fig. 3 theoretical EAGB isochrones (from Bertelli et al. 1994) are overlaid on the HST CMD of the post-starburst BCD galaxy, VII Zw 403, and we can see that a large population of EAGB stars is a bright indicator of a past high $s f r$, and the luminosity spread depends upon metallicity and the age of the event.

\subsection{Distance, Extinction \& Metallicity}

The accurate interpretation of the indicators described above depends critically upon having reliable estimates for the distance, the extinction and the metallicity of a galaxy. Ideally we would also like to know if the extinction is patchy and on what scale, and what has been the evolution of the metallicity of the stellar population with time. These three basic parameters, in conjunction with observational errors and incompleteness, make the most significant impact on the properties of the CMD and hence the final SFH model (Tolstoy et al. 1998). There are a number of difficulties in accurately determining these basic properties but they can be resolved with careful observation and analysis techniques. 
The Distance is the most crucial parameter for accurate analysis of a CMD, partly because it can easily be wrong by many orders of magnitude (for example the young, red supergiants can be mistaken for the RGB, if the observed CMD isn't deep enough to confirm the identification, i.e. by detecting a $\mathrm{RC}$ or $\mathrm{HB}$ ). If the distance to a galaxy is incorrect this will result in the assumed ages of the different populations being completely wrong. To be sure of the distance to small faint galaxies it is necessary to have a CMD which goes deep enough to extend below the unambiguous RC/HB structure (Tolstoy et al. 1998).

The Extinction, both internal to a galaxy and between us and a galaxy, can affect the accurate analysis of a CMD. If the extinction is incorrectly determined it will have the same effect as a distance error, and hence affect the reliability of the SFH models (e.g., Gallagher et al. 1998).

Metallicity: When a galaxy makes stars, then the detritus of this process (e.g., from SN explosions and stellar winds) make it unlikely that the galaxy can avoid metallicity evolution altogether. However, there is no concrete observational evidence that this is true, although abundance ratios of different elements do give us model dependent suggestions (Pagel 1994). Determining the effect of metallicity evolution in a CMD is difficult. It is impossible to determine a unique model based solely on the RGB because of age-metallicity degeneracy, and if metallicity evolution is neglected then the best model for that galaxy will typically be younger than if metallicity evolution were included (e.g., Tolstoy et al. 1998).

\subsection{High Quality Imaging}

Typically the prime limiting factor in making an accurate analysis of a CMD is image crowding, due to overlapping stellar images. There is basically no way to "correct" for crowding in a CMD, so all that can be done is to estimate the size of the uncertainties that result from crowding, from incompleteness analysis (e.g., Tolstoy 1996), and apply these to any results from the interpretation of the CMD. Using HST significantly reduces crowding problems, and in certain cases, such as dwarf irregular galaxies, excellent seeing from the ground will also be sufficient to overcome severe crowding problems.

\section{The Data: Recent HST Observations}

Thus the time is ripe for re-observing the resolved stellar populations of all the galaxies in our LG. Much of our detailed knowledge of the SFHs of galaxies beyond 1 Gyr ago comes from the Milky Way and its nearby dSph satellites or from HST CMDs. To date, the limiting factors have been crowding and resolution limits for accurate stellar photometry from the ground. The large collecting area, the field of view, and the extremely impressive image quality and stability of VLT UT1, combined with the extremely good seeing attainable at Paranal, makes it possible to obtain more accurate CMDs to fainter magnitudes, (i.e. do better) than HST. These facilities provide unique opportunities to extend beyond our immediate vicinity and encompass the whole LG. To date HST has observed the resolved stellar populations in a variety of nearby galaxies (e.g., dE: NGC 147, Han et al. 1997; Irr: LMC, Geha et al. 1998; Spiral: M 31, Holland et al. 1996; M 33, Sarajedini et al. 1998; BCD: VII Zw 403, Lynds et al. 1998; 
NGC 1569, Greggio et al. 1998; dI: Leo A Tolstoy et al. 1998; dSph: Leo I, Gallart et al. 1999). From every galaxy which has been looked at carefully something new has been learnt.

An HST program was initiated by Skillman (e.g., Skillman et al. 1998), observing a sample of four nearby dI galaxies (Sextans A, Pegasus, Leo A \& GR 8), using four orbits of telescope time per galaxy, in three filters (effectively $B V I$ ). The results have been dramatic and illustrate the tremendous advances possible, even with short exposures, when crowding has been virtually illuminated. Here I am going to provide a summary of this program.
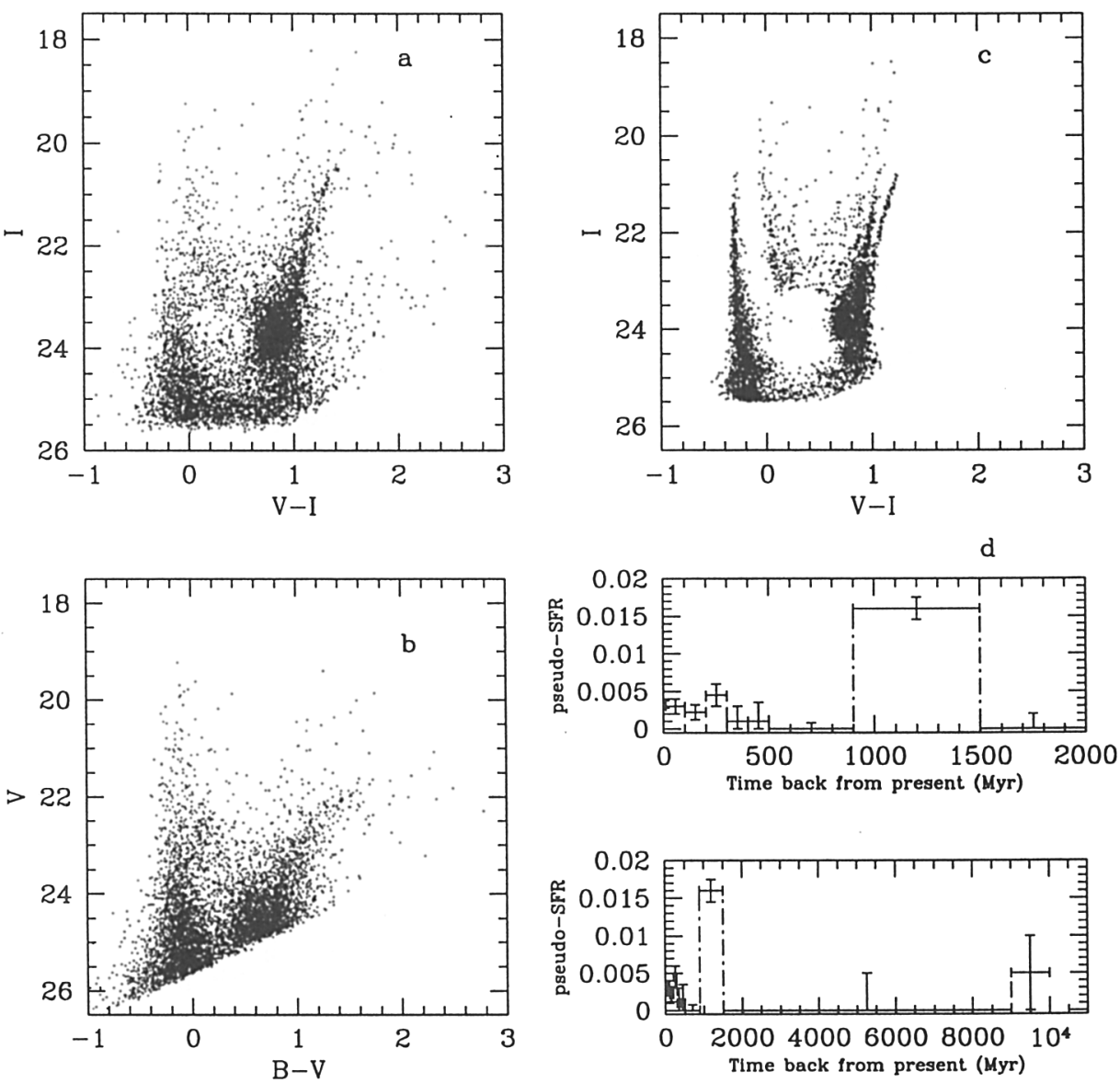

Figure 4. Here we show the results for the analysis of the HST/WFPC2 data of Leo A (Tolstoy et al. 1998). In (a) is the $V-I, I$ CMD, 1 orbit exposure time per filter. In (b) is the $B-V, V$ CMD, 2 orbits in $B$. In (c) is the best match Monte-Carlo simulation model (in $V-I, I$ ) found for these data convolved with the theoretical measurement error distribution, and in (d) is the SFH that created the model CMD which best matches these data. See Tolstoy et al. 1998 for more details. 


\subsection{Leo A}

Leo A (三 DDO 69, Leo III, UGC 5364) is a nearby gas-rich Magellanic dI galaxy. Analysis of the WFPC2 CMDs of the resolved stellar population (see Fig. 4) enabled a new distance and an accurate SFH to be determined for this extremely metal-poor galaxy (Tolstoy et al. 1998). From the position of the RC, BLs and the tip of the RGB, a distance modulus, $m-M=24.2 \pm 0.2$, or $690 \pm 60 \mathrm{kpc}$, was obtained which places Leo A firmly within the LG. The interpretation of the WFPC2 CMDs at this new distance, based upon extremely low metallicity $(Z=0.0004)$ theoretical stellar evolution models, suggests that this galaxy is predominantly young, i.e. $<2$ Gyr old. A major episode of star formation 900-1500 Gyr ago can explain the RC luminosity and also fits in with the interpretation of the number of anomalous Cepheid variable stars seen in this galaxy. The presence of an older, underlying globular cluster-age stellar population could not be ruled out with these data. However, using the currently available stellar evolution models, it would appear that such an older population is limited to no more than $10 \%$ of the total star formation to have occurred in the centre of this galaxy. Using theoretical models of the chemical evolution of dwarf galaxies (Ferrara \& Tolstoy 1999) however, it is clear that even though this galaxy is extremely metal poor in order for it to build up the current metallicity it must contain a significant underlying older stellar population, perhaps in an extended outer halo of older stars. Of course neither the chemical evolution models nor the existing CMDs can distinguish between an old population which formed in a large burst, or at a more sedate and roughly constant rate throughout a longer time.

\subsection{Pegasus}

The Pegasus dI galaxy is a gas-rich system in the LG, it is possibly an example of a transition object between a dwarf galaxy dominated by current star-formation and one dominated by past star formation. Analysis of the WFPC2 CMD of the Pegasus dI (see Fig. 5) reveals a young main sequence (MS), a well populated RGB, a small number of EAGB stars and near the faint limits of the data a populous RC (Gallagher et al. 1998). The young stellar component is clustered in two centrally-located clumps, while older stars form a more extended disk or halo. The colours of the MS require a relatively large extinction $\left(\mathrm{A}_{V}=0.47\right.$ $\mathrm{mag}$ ), and the (extinction corrected) mean colour of the well-populated RGB is relatively blue, consistent with a moderate metallicity young, or older and more metal-poor stellar population. The distance of Pegasus was revised to be $760 \mathrm{kpc}$, taking account of the higher reddening. The RGB has significant width in colour, implying a range of stellar ages and/or metallicities. A SFH which was consistent with the data is one in which the $s f r$ was higher, by a factor of 3-4, about $1 \mathrm{Gyr}$ ago. It was impossible to constrain the SFH beyond 1 Gyr ago, as seen by the large error bars in Fig. $5 \mathrm{~d}$, without better information on stellar metallicities and deeper photometry. The youngest model consistent with the data contains stars with constant metallicity of $\mathrm{Z}=0.001$ which mainly formed 2-4 Gyr ago. If stellar metallicity declines with increasing stellar age, then the older ages extend up to $\sim 8$ Gyr. However, even at its peak of star forming activity, the intermediate-age dominated model for the Pegasus dwarf most likely remained relatively $\operatorname{dim}$ with $\mathrm{M}_{V} \approx-14$. 

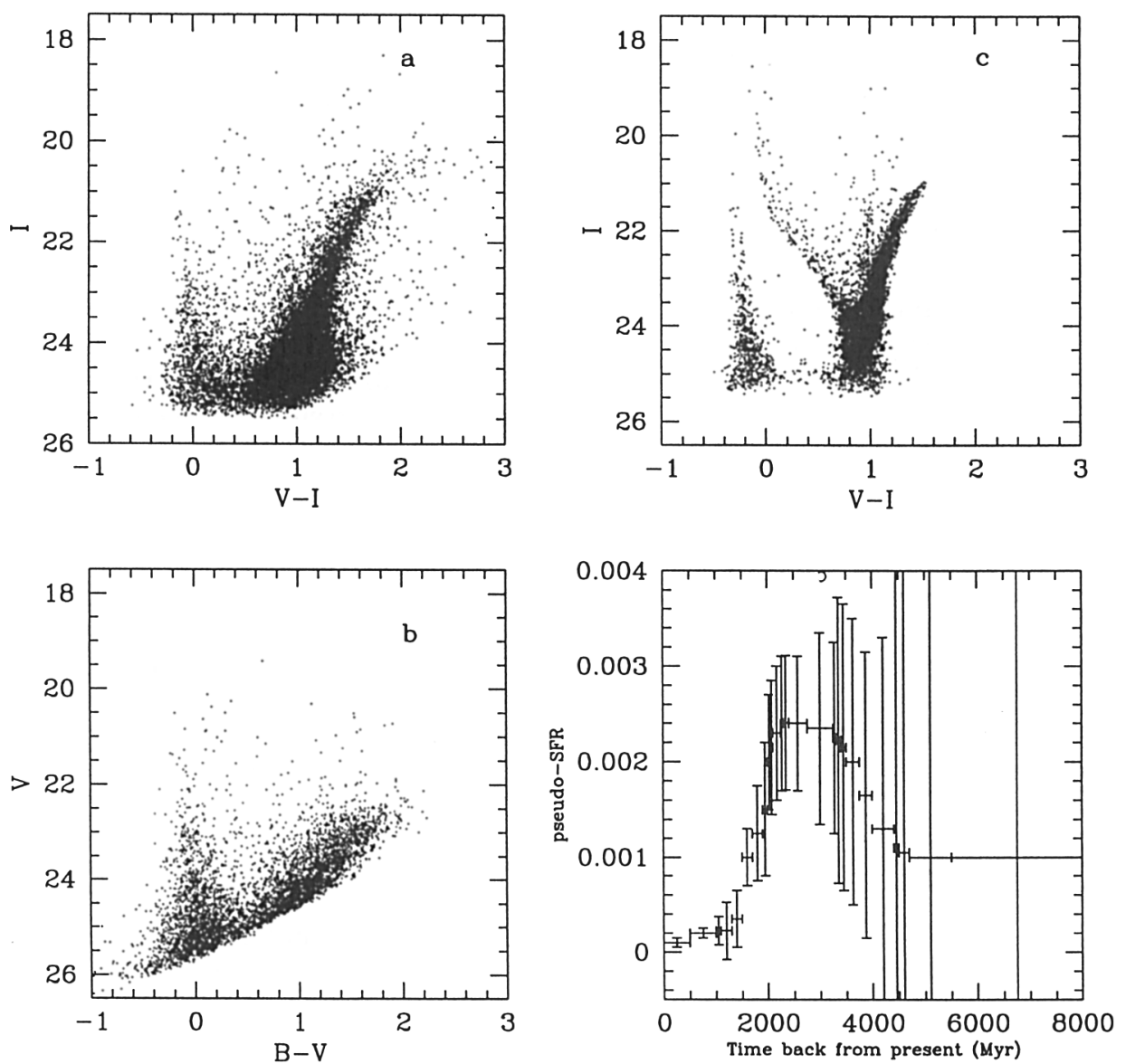

Figure 5. Here we show the results for the HST/WFPC2 analysis of Pegasus (Gallagher et al. 1998). In (a) is the $V-I, I$ CMD, 1 orbit exposure time per filter. In (b) is the $B-V, V$ CMD, 2 orbits in $B$. In (c) is the best match Monte-Carlo simulation model, in $V-I, I$, found for these data (excluding the $R C$ ) and convolved with the theoretical measurement error distribution, and in (d) is the SFH that created the model CMD which best matches the data. See Gallagher et al. 1998 for more details.

\subsection{Sextans A}

Sextans A (DDO 75, A 1008-04) is a gas-rich dI galaxy with active star formation, which is located on the periphery of the Local Group at a distance of 1.44 Mpc. The CMD of Sextans A (Fig. 6) shows several clearly separated populations that align well with stellar evolution model predictions for a low metallicity system (Dohm-Palmer et al. 1997b). The SFH from the MS+BL stars was determined over the last $600 \mathrm{Myr}$. This is the first time a BL sequence has been clearly identified in a CMD. The distribution of the BL stars was combined with 
sfr calculations to determine the spatial variation of the star formation across Sextans A with time. The modelling concludes that in the past $50 \mathrm{Myr}$, Sextans A has had an average $s f r$ that is $\sim 10$ times that of the average $s f r$ over the history of the galaxy. This current activity is highly concentrated in a young region in the Southeast roughly 25 pc across. This coincides with the brightest $\mathrm{H}$ II regions and the highest column density of $\mathrm{H}$ I. This one region contains half of all the current star formation activity within the field of view. Between the ages of 100 and $600 \mathrm{Myr}$ ago, the star formation has been roughly constant at slightly above the average value. There are regions (200-300pc across) with a factor of $\sim 5$ enhancement in $s f r$ with a duration of 100-200 Mvr.
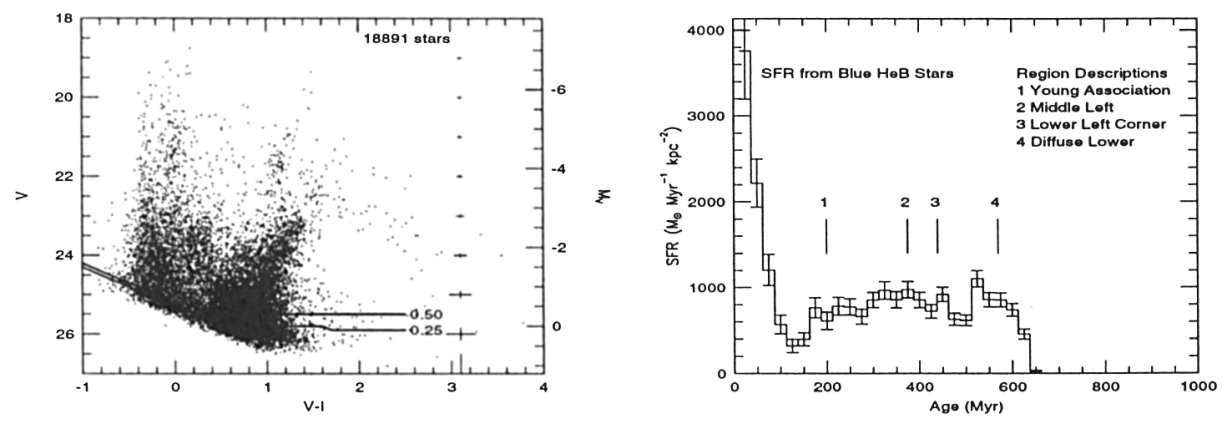

Figure 6. Here we show the results for the HST/WFPC2 analysis of Sextans A (Dohm-Palmer et al. 1997b). On the left is the $V-I, I$ CMD, 1 orbit exposure time per filter. On the right is the SFH from the MS+BL which best matches the data. See Dohm-Palmer et al. for more details.

\section{4. $\quad$ GR 8}

The final galaxy looked at with WFPC2 in this sample is GR 8 (DDO 155, UGC 8091), a gas-rich dI galaxy with active star formation, on the edge of the LG at a distance of $2.2 \mathrm{Mpc}$ (Dohm-Palmer et al. 1998). It is slightly more compact and distant than the other galaxies in the Skillman sample, and so crowding was more of a problem in the analysis of the WFPC2 data. Artificial star tests showed the data to be $50 \%$ complete to $V=26.3, B=25.4$, and $I=25.2$. The CMD (Fig. 7) shows a well defined population with a very young MS (<10 Myr), and an RGB as old as several Gyr. A distance estimate, based on the tip of the RGB, was found to be in excellent agreement with the Cepheid determination of $\mu=26.75 \pm 0.35$, or $2.2 \mathrm{Mpc}$ (Tolstoy et al. 1995). There is also evidence for an extended stellar "halo" beyond the $\mathrm{HI}$ distribution. Based on the MS and BL luminosity function the $s f r$ over the past $500 \mathrm{Myr}$ was estimated to have been fairly constant, with up to $60 \%$ variations. The star formation appears to occur in super-association size regions (100-200 pc across), which last $\sim 100 \mathrm{Myr}$. These regions come and go with no obvious pattern, except that they seem to concentrate in the current locations of $\mathrm{H}$ I clumps. This suggests 
that the $\mathrm{H}$ I clumps are long-lived features that support several star forming events over time.
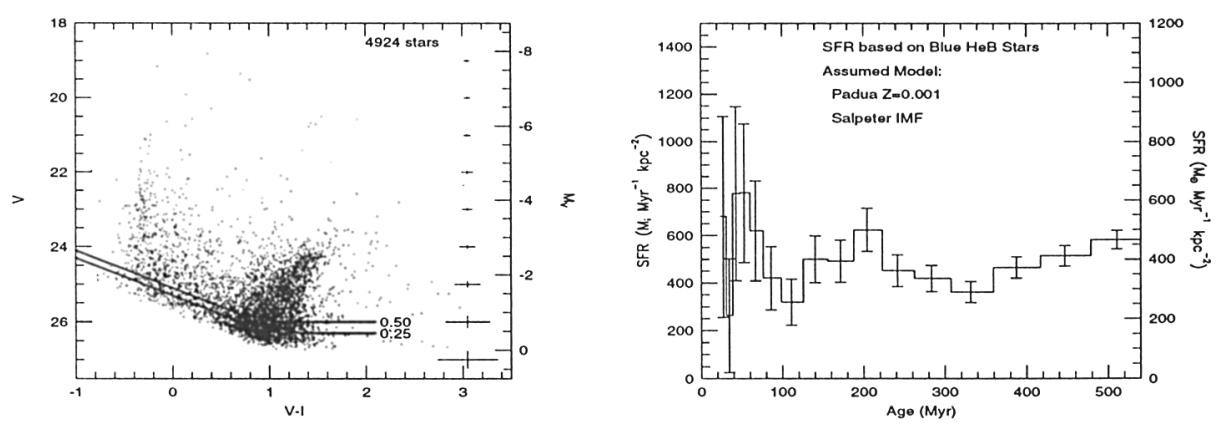

Figure 7. Here we show the results for the HST/WFPC2 analysis of GR 8 (DohmPalmer et al. 1998). On the left is the $V-I, I$ CMD, 1 orbit exposure time per filter. On the right is the SFH from the MS+BL which best matches the data. See Dohm-Palmer et al. for more details.

\section{The Scenario}

There appear to be a diversity of star formation properties between apparently similar galaxy types. This appears to correlate with neither the metallicity of a galaxy nor its gas content. In this sample there is no direct evidence (MSTOs) for any discrete strong bursts of star formation. Comparing the results of the four dI galaxies in the HST sample of Skillman: Sextans A, Pegasus DIG, Leo A, \& GR 8, a trend of higher $s f r$ per area with larger $M_{\mathrm{HI}} / L_{B}$ was found (DohmPalmer et al. 1998). Although Pegasus and Leo A have clearly had higher global star formation rates in the past, whether this took place over several Gyr or in a short discrete burst cannot be discerned with these data. Leo $\mathrm{A}$ is the best nearby candidate for a primeval galaxy. If there was an epoch during which galaxies had frequent bursts it was beyond $z=0.1$. Deeper observations of more galaxies will push this limit back in redshift, and make the connection to the faint blue galaxy population more convincing. The giant galaxies (M 31, the Galaxy, M 33) have large old populations, and must contain the bulk of star formation in the LG since they contain something like $90 \%$ of the stellar mass, but this does not mean that they have always dominated in luminosity as completely as they do now. This is why it is important to look for detailed evidence of bursting behaviour in the most common (by number) galaxies in the LG - the late type irregular galaxies - by looking at the fossil record of the old/intermediate age resolved stellar populations.

Acknowledgements. I gratefully acknowledge the organisers for a generous grant, and I also thank my collaborators on the "HST dwarf galaxy consortium", namely, Evan Skillman, Robbie Dohm-Palmer, Jay Gallagher, Andrew Cole, Mario Mateo, Abi Saha \& John Hoessel who have been involved in all of the projects I have reported on here. 


\section{References}

Aparicio, A., Gallart, C., Chiosi, C., Bertelli, G. 1996, ApJ, 469, L97

Bertelli, G., Bressan, A., Chiosi, C., Fagotto, F., Nasi, E. 1994, A\&AS, 106, 275 Caputo, F., et al. 1995, A\&A, 304, 365

Cole, A. 1998, ApJ, 500, L137

Da Costa, G.S. 1997, in: Stellar Astrophysics for the Local Group: A First Step to the Universe, (eds.) A. Aparicio \& A. Herrero, p. 351

Dohm-Palmer, R.C., Skillman, E.D., Saha, A., Tolstoy, E., Mateo, M., Gallagher, J.S., Hoessel, J., Chiosi, C., Dufour R. 1997a, AJ, 114, 2527

Dohm-Palmer, R.C., Skillman, E.D., Saha, A., Tolstoy, E., Mateo, M., Gallagher, J.S., Hoessel, J., Chiosi, C., Dufour, R. 1997b, AJ, 114, 2514

Dohm-Palmer, R.C., Skillman, E.D., Saha, A., Tolstoy, E., Mateo, M., Gallagher, J.S., Hoessel, J., Chiosi, C., Dufour, R. 1998, AJ, 116, 1227

Fagotto, F., Bressan, B., Bertelli, G., Chiosi, C. 1994, A\&AS, 104, 365

Ferrara, A., Tolstoy, E. 1999, to be submitted

Fukugita, M., Hogan, C.J., Peebles, P.J.E. 1998, ApJ, 503, 518

Gallagher, J.S., Tolstoy, E., Saha, A., Hoessel, J., Skillman, E.D., Dohm-Palmer, R.C., Mateo M. 1998, AJ, 115, 1869.

Gallart, C., Aparicio, A., Chiosi, C., Bertelli, G., Vilchez, J. 1994, ApJ, 425, L9

Gallart, C., et al. 1999, ApJ, in press (astro-ph/9811122)

Geha, M.C., et al. 1998, AJ, 115, 1045

Greggio, L., et al. 1998, ApJ, 504, 725

Han, M., et al. 1997, AJ, 113, 1001

Holland, S., et al. 1996, AJ, 112, 1035

Hurley-Keller, D., Mateo, M., Nemec, J. 1998, AJ, 115, 1840

Lynds, R., Tolstoy, E., O'Neil, E., Hunter, D.A. 1998, AJ, 116, 146

Madau, P., Pozzetti, L., Dickinson, M. 1998, ApJ, 498, 106

Mateo, M. 1998, ARA\&A, 36, 435

Mighell, K. 1997, AJ, 114, 1458

Pagel, B. 1994, in: The Formation and Evolution of Galaxies, (eds.) C. MuñozTuñóu \& F. Sánchez, CUP, p. 149

Sarajedini, A., Geisler, D., Harding, P., Schommer R. 1998, ApJ, 508, L37

Skillman, E., et al. 1998, in: The Magellanic Clouds and other Dwarf Galaxies, (eds.) T. Richtler \& J.M. Braum, p. 77

Tolstoy, E. 1996, ApJ, 462, 684

Tolstoy, E. 1998, in: Dwarf Galaxies \& Cosmology, (eds.) Thuan et al., in press (astro-ph/9807154)

Tolstoy, E., et al. 1995, AJ, 109, 579

Tolstoy, E., Saha, A. 1996, ApJ, 462, 672

Tolstoy, E., Gallagher, J.S., Cole, A.A., Hoessel, J., Saha, A., Dohm-Palmer,

R.C., Skillman, E.D., Mateo, M., Hurley-Keller, D. 1998, AJ, 116, 1244

Tosi, M., Greggio, L., Marconi, G., Focardi, P. 1991, AJ, 102, 951 


\section{Discussion}

Lynden-Bell: You implied that you would reject models with a lower metal abundance at a later time, but if a galaxy accreted gas from outside is that not possible?

Tolstoy: (The question refers to VII ZW 403) I agree that this is indeed possible. But for me to make such a radical statement I would need to trust the models better upon which this is based. EAGB models are notoriously uncertain.

Geisler: Why does the upper giant branch become much bluer at lower spatial resolution (i.e. crowding effect), and why isn't the lower GB as affected?

Tolstoy: There is no information on the lower GB. I presume the effect would be similar.

Aparicio: You said that the stars between the RGB and the MS in Pegasus are likely to be the result of differential reddening and also that they could be a blue-loop population. I personally prefer the second interpretation because: (1) their number is compatible with a blue-loop population; (2) the reddening should be large and in that case, its effects would be evident around the RGB, but they are not.

Tolstoy: I believe the stars between the RGB and the MS in Pegasus are blueloop stars affected by reddening.

Hesser: I'm a bit puzzled by your statement regarding absence of binaries, when Carme Gallart's modelling implies high binary frequency in other systems. Can either of you help me understand better the apparent discrepancy here?

Tolstoy: Gallart's findings for Leo I are for a population of stars much further down the main sequence and therefore much more affected by binaries. Because I don't need to assume a binary fraction larger than 10-20\% I don't. Depth effects (thick galaxy along the line of sight) also can broaden the main sequences considerably (cf. SMC) of very nearby galaxies.

Cannon: A concern in interpreting the CMDs is the possible direct influence of binary stars, not only unresolved binaries giving peculiar positions as Gallart has found, but also interacting binaries affecting the stellar evolution. In particular, interpreting the blue loops may depend critically on the incidence of binarity, as shown by the difficulties encountered in understanding supernova $1987 \mathrm{~A}$ in the LMC.

Tolstoy: Agreed. In our dwarf irregular (upper, or young main sequence/blue loop) analysis we see no evidence for this. Typically the blue loop models match the data surprisingly well! Both in numbers of stars and positions, except in Pegasus where there is a problem with reddening issues. But it is an issue we always have to bear in mind. 